\title{
New Stabilization Properties of Pendulum Models Applying a Large Parameter
}

\author{
A. I. Ismail (iD ${ }^{1,2}$ and Hamza A. Ghulman ${ }^{1}$ \\ ${ }^{1}$ Mechanical Engineering Department, College of Engineering and Islamic Architecture, Umm Al-Qura University, \\ P.O. Box 5555, Makkah, Saudi Arabia \\ ${ }^{2}$ Mathematics Department, Faculty of Science, Tanta University, P.O. Box 31527, Tanta, Egypt \\ Correspondence should be addressed to A. I. Ismail; aiismail@uqu.edu.sa
}

Received 12 November 2021; Revised 15 December 2021; Accepted 23 December 2021; Published 18 January 2022

Academic Editor: Viet-Thanh Pham

Copyright (c) 2022 A. I. Ismail and Hamza A. Ghulman. This is an open access article distributed under the Creative Commons Attribution License, which permits unrestricted use, distribution, and reproduction in any medium, provided the original work is properly cited.

\begin{abstract}
In the present paper, we introduce new models of pendulum motions for two cases: the first model consists of a pendulum with mass $M$ moving at the end of a string with a suspended point moving on an ellipse and the second one consists of a pendulum with mass $M$ moving at the end of a spring with a suspended point on an ellipse. In both models, we use the Lagrangian functions for deriving the equations of motions. The derived equations are reduced to a quasilinear system of the second order. We use a new mathematical technique named a large parameter method for solving both models' systems. The analytical solutions are obtained in terms of the generalized coordinates. We use the numerical techniques represented by the fourth-order Runge-Kutta method to solve the autonomous system for both cases. The stabilities of the obtained solutions are studied using the phase diagram procedure. The obtained numerical solutions and analytical ones are compared to examine the accuracy of the mathematical and numerical techniques. The large parameter technique gives us the advantage to obtain the solutions at infinity in opposite with the famous Poincare's (small parameters) method which was used by many outstanding scientists in the last two centuries.
\end{abstract}

\section{Introduction}

The pendulum motions are considered as one of the important problems in theoretical mechanics. These problems are studied by many authors in [1-5]. The authors used the small parameter technique for solving their problems. In [6], the author studied the properties of the relative periodic motions of a coherent object suspended by a flexible wire at a regular rotating vertical plane. He used Lagrange's equations to obtain the motion equations while neglecting nonlinear boundaries. He found periodic solutions to equations using the small parameter method. In [7], the movement of a variable-length pendulum was studied and perturbation analysis [8] was used to determine the properties of the movement. In [9], the authors studied a simple pendulum revolving around an axis that has a double linear torque and is subject to periodic movements. The researchers showed that this natural system becomes an effective way to determine the change in the median resonance (parametric). In [10], the author concluded solutions for a simple pendulum in the presence of excitation in the polar direction.

Nobody thought about using another technique especially the large parameter method although this technique allows us to give the problem new conditions that cannot be assumed previously. Also, this technique gives us the chance to study the problems in a new domain of the problem (at infinity). The applied work has many applications in the rotary planet motions around the Sun and the rotary motions of bodies around the Earth. Also, there are many applications in satellite motions, antennas, and navigations. In the first problem, the angular velocity $\omega$ is the one for the point $Q$ instead of point $\mathrm{A}$ in the previous works (see Figure 1). Also, the angle $\omega t$ is the angle between the line OQ and the fixed vertical downward $x$-axis in the plane xy instead of the angle between the line OA and the $x$-axis. However, in the second problem, we take a rigid body $M$ 


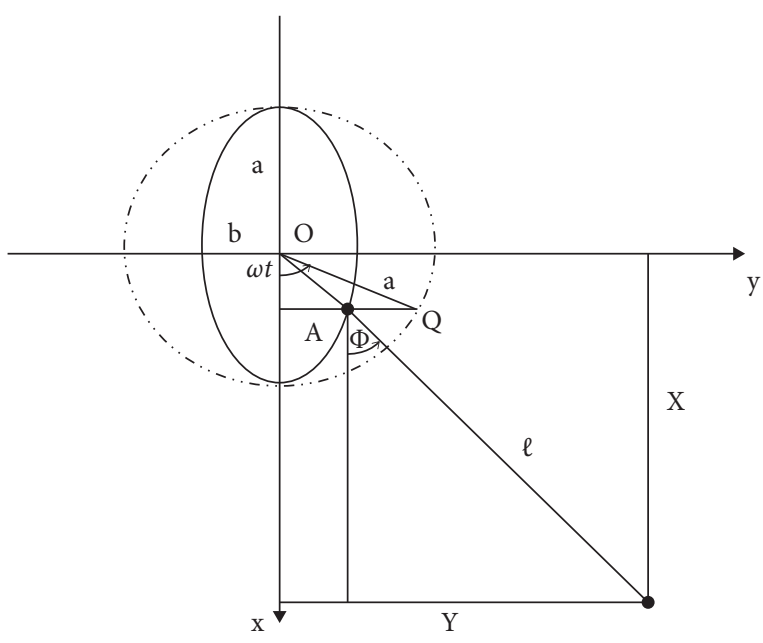

FIGURE 1: The pendulum motion on an ellipse.

instead of a particle in the first one and spring instead of a string in the first. So, the second problem takes a complicated study and procedure for obtaining the solutions in terms of three generalized coordinates instead of one in the first problem. We take into consideration the mentioned descriptions above for studying the following two models and constructing the equations of motions using the Lagrangian function. We achieve the solutions by the large parameter method instead of the small one. This method is considered as a new procedure [11] which gives new considerations for these problems.

\section{The First Pendulum Model}

In this section, we study a pendulum of mass $M$ and string length $\ell$ with suspended point A moving on an ellipse (see Figure 1). For this case, we take a point $Q$ on the auxiliary circle of radius $a$ to correspond the point A on the ellipse. Let $\mathrm{O}$ be the common center of both the ellipse and the circle such that the line AQ is perpendicular on the major axis of the ellipse. When point A moves on the ellipse, the point $Q$ moves on the circle with angular velocity $\omega$ in the plane $x y$. Let $\mathrm{A}$ begin the motion at the initial moment $t=0$ in an anticlockwise direction. After a time $t$, the coordinates of the point A becomes $\left(x_{A}, y_{A}\right)$ such that

$$
\omega t=\cos ^{-1}\left(\frac{x_{A}}{a}\right)=\sin ^{-1}\left(\frac{y_{A}}{b}\right),
$$

where $2 a$ and $2 b$ are the major and the minor axes of the ellipse, respectively. The coordinates $(X, Y)$ of $m$ are obtained as

$$
\omega t=\cos ^{-1}\left[\frac{(X-\ell \cos \Phi)}{a}\right]=\sin ^{-1}\left[\frac{(Y-\ell \sin \Phi)}{b}\right],
$$

where $\Phi$ is the angle between the string and the vertical axis. Assume the following parameters [12],

$$
\begin{aligned}
& \mu=\frac{b}{\ell}>>1, \\
& a=b v,
\end{aligned}
$$

where $\mu$ is a large parameter, that is, $\ell<<b$.

Also, we assume the variables

$$
\begin{aligned}
\ell \omega_{n}^{2} & =g, \\
\omega \Omega & =\omega_{n}, \\
\Phi & =\mu^{-1} \varphi, \\
\frac{\tau}{\omega} & =t,
\end{aligned}
$$

where $g$ is the gravity of acceleration, $\omega_{n}$ is the normal angular velocity, and $\varphi$ is the generalized coordinate for the problem.

2.1. Equation of Motion. Using Lagrange's equation, we get the equation of motion of the pendulum in the form as follows:

$$
\frac{\mathrm{d}}{\mathrm{d} \tau}\left[\frac{\partial(T-V)}{\partial \varphi^{\prime}}\right]-\left[\frac{\partial(T-V)}{\partial \varphi}\right]=0,
$$

where $T$ is the kinetic energy and $\mathrm{V}$ is the potential one. Substituting (2), (3), and (4) into (5), we get

$$
\begin{aligned}
\varphi^{\prime \prime}+\Omega^{2} \varphi= & \sin \tau-\mu^{-1} \nu \varphi \cos \tau-0.5 \mu^{-2} \varphi^{2} \sin \tau \\
& +0.17 \mu^{-3} \nu \varphi^{3} \cos \tau .
\end{aligned}
$$

The solution of this equation means that we obtain $\varphi$ in terms of the large parameter and the time.

2.2. Approximate Periodic Solution. Now, we will find the approximated periodic solutions for the nonresonance case [12]; that is $\Omega$ is irrational value. However, here we use the large parameter technique instead of the small one which was used previously. The solutions of (6) are obtained in the form of power series expansions of powers of $1 / \mu$ as follows:

$\varphi(\tau, \mu)=\varphi_{0}(\tau)+\mu^{-1} \varphi_{1}(\tau)+\mu^{-2} \varphi_{2}(\tau)+\mu^{-3} \varphi_{3}(\tau)+\ldots$

Substituting from (7) into (6) and equating coefficients of like powers of $(1 / \mu)$ of both sides, we get a system of differential equations containing $\varphi_{i}, i=1,2,3, \ldots$, which is solved to obtain the following: 


$$
\begin{aligned}
\left(\Omega^{2}-1\right) \varphi(\tau, \mu)= & \sin \tau-\mu^{-1} \frac{\nu}{\left(\Omega^{2}-4\right)} \sin \tau \cos \tau \\
& +\mu^{-2}\left[\frac{2 \nu^{2}\left(\Omega^{2}-1\right)-3\left(\Omega^{2}-4\right)}{8\left(\Omega^{2}-1\right)^{2}\left(\Omega^{2}-4\right)} \sin \tau+\frac{2 \nu^{2}\left(\Omega^{2}-1\right)+\left(\Omega^{2}-4\right)}{8\left(\Omega^{2}-1\right)\left(\Omega^{2}-4\right)\left(\Omega^{2}-9\right)} \sin 3 \tau\right] \\
& +\nu \sin 2 \tau \mu^{-3}\left[\begin{array}{l}
\frac{\left(5 \Omega^{4}-62 \Omega^{2}+123\right)-3 \nu^{2}\left(\Omega^{2}-1\right)\left(\Omega^{2}-5\right)}{12\left(\Omega^{2}-1\right)^{2}\left(\Omega^{2}-4\right)^{2}\left(\Omega^{2}-9\right)} \\
-\frac{\left(5 \Omega^{4}-44 \Omega^{2}+51\right)+3 \nu^{2}\left(\Omega^{2}-1\right)^{2}}{12\left(\Omega^{2}-1\right)^{2}\left(\Omega^{2}-4\right)\left(\Omega^{2}-9\right)\left(\Omega^{2}-16\right)} \cos 2 \tau
\end{array}\right]+\cdots
\end{aligned}
$$

\section{The Second Pendulum Model}

In this section, we consider a rigid body pendulum of mass $M$ suspended with a massless spring with a length $\rho(t)$ which is suspended at a point $O_{1}$ on the ellipse [3]. According to [3], the point $Q$ moves on the auxiliary circle with constant angular velocity $\omega$ and corresponds to point $O_{1}$ on the ellipse. Consider that the circle has radius $b$ and the angle between the line $O Q$ and the horizontal axis depends on $t$ only. Consider the motion in the plane $x y$. From [3], the point $O_{1}$ moves from $t=0, \theta=0$, and $\varphi=0$ in the counterclockwise direction.

3.1. Determining of Lagrangian Function. After a while $t$, the point $O_{1}$ will create an angle $(\omega t)$ with the horizontal axis, that is:

$$
\omega t=\cos ^{-1}\left(\frac{x_{O_{1}}}{a}\right)=\sin ^{-1}\left(\frac{y_{O_{1}}}{b}\right),
$$

where $2 a$ and $2 b$ are the minor and major diagonal of the ellipse, respectively.

Consider that the coordinates of the center of mass of the body are given by

$$
\begin{aligned}
& X_{c}=a \cos \omega t+\rho \sin \theta+h \sin \varphi, \\
& Y_{c}=b \sin \omega t+\rho \cos \theta+h \cos \varphi .
\end{aligned}
$$

We calculate the velocity of the center $c$ by differentiating (9) and (10). Consider the following parameters and variables [3]:

$$
\begin{aligned}
\mu & =\frac{h}{d}>>1, \\
\rho(t) & =d+\beta(t), \\
\omega_{n}^{2} & =\frac{K}{M \omega^{2}}, \\
J \sigma^{2} & =M h\left(\frac{g}{\omega^{2}}+\gamma\right), \\
\gamma \omega^{2} & =\frac{K(d-l)}{M-g}, \\
\theta & =\mu^{-1} \Theta, \quad(\theta \varphi),
\end{aligned}
$$

where $d$ is the spring length at relative equilibrium, $J$ denotes the principal moment of inertia for the axis $c \zeta, l$ is the free length of the spring, $\mu$ is a large parameter, $\mathrm{K}$ is the force constant of the spring, and $\beta, \Theta$, and $\Phi$ are the generalized coordinates. Use (9), (10), (11), and (13) to find the kinetic energy and potential one and then construct the Lagrangian = kinetic energy-potential one in terms of the generalized coordinates as follows: 


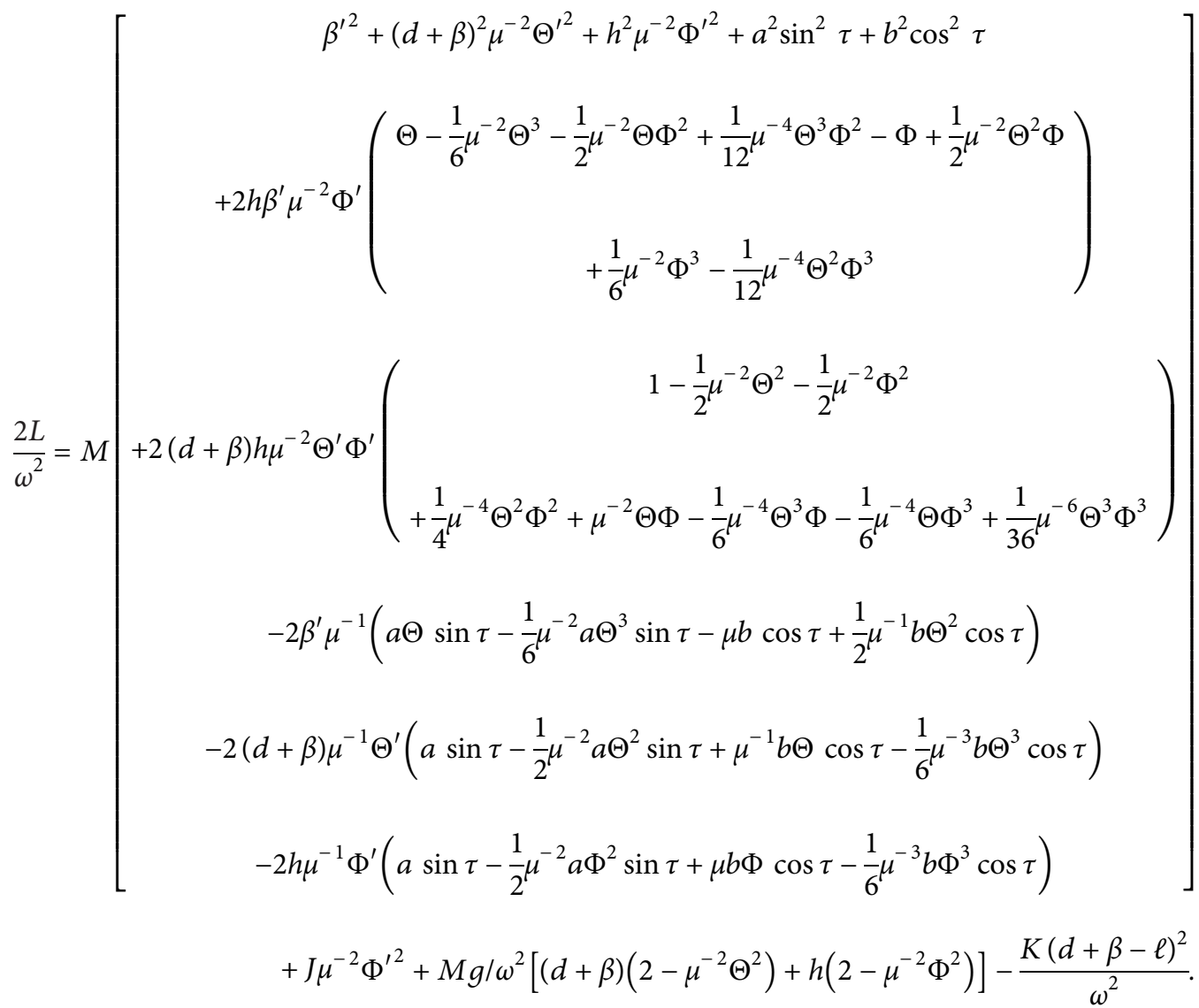

3.2. Equations of Motion. Making use of Lagrange's equations (14), (11), and (13), we get the equations of motion as follows:

$$
\begin{aligned}
\beta^{\prime \prime}+\omega_{n}{ }^{2} \beta= & b \sin \tau-\gamma+\mu^{-1}\left[a \Theta \cos \tau+h \Theta^{\prime 2}\right] \\
& +\mu^{-2}\left[h\left(\Phi^{\prime \prime} \Phi-\Phi^{\prime \prime} \Theta+\Phi^{\prime 2}\right)+\beta \Theta^{\prime 2}+0.5 \Theta^{2}\left(\frac{g}{\omega^{2}}-b \sin \tau\right)\right], \\
h \Theta^{\prime \prime}= & a \cos \tau-\mu^{-1}\left[2 \beta^{\prime} \Theta^{\prime}+\Theta\left(b \sin \tau+\frac{g}{\omega^{2}}\right)+h \Phi^{\prime \prime}\right]-0.5 a \mu^{-2} \Theta^{2} \cos \tau \\
\mu^{-1}\left[\left(h+\frac{J}{M h}\right) \Phi^{\prime \prime}+\frac{g}{\omega^{2}} \Phi\right]= & a \cos \tau-h \Theta^{\prime \prime}+\mu^{-1}\left[-2 \beta^{\prime} \Theta^{\prime}-\beta \Theta^{\prime \prime}+\beta^{\prime \prime} \Phi-b \Phi \sin \tau\right] \\
& +\mu^{-2}\left[-\frac{1}{2} a \Phi^{2} \cos \tau+\frac{1}{2} h \Theta^{\prime \prime}\left(\Theta^{2}+\Phi^{2}\right)-h\left(\Theta^{\prime \prime} \Theta \Phi-\Theta \Theta^{\prime 2}+\Theta^{\prime 2} \Phi\right)\right] \\
& +\mu^{-3}\left[(\Theta-\Phi)^{2}\left(\beta^{\prime} \Theta^{\prime}+\frac{1}{2} \beta \Theta^{\prime \prime}\right)+\beta \Theta^{\prime 2}(\Theta-\Phi)+\frac{1}{6} \beta^{\prime \prime}(\Theta-\Phi)^{3}+\frac{1}{6} b \Phi^{3} \sin \tau\right] .
\end{aligned}
$$

Substituting (15) and (16) into (17), we get 


$$
\left(\Phi^{\prime \prime}+\sigma^{2} \Phi\right) J=M h\left[\begin{array}{c}
\Theta\left(a \Theta \cos \tau+h \Theta^{\prime 2}\right) \\
+\mu^{-2}\left[\begin{array}{c}
\left.h \omega^{2}+b \sin \tau\right)-\omega_{n}^{2} \beta \Phi+\mu^{-1}\left[\begin{array}{c} 
\\
+0.5 a \cos \tau\left(\Theta^{2}-\Phi^{2}\right)+0.5 h \Theta^{\prime \prime}(\Theta-\Phi)^{2}+h \Theta^{\prime 2}(\Theta-\Phi)
\end{array}\right] \\
+\frac{g}{\omega^{2}} \Theta^{2} \Phi+\beta^{\prime} \Theta^{\prime}(\Theta-\Phi)^{2}+0.5 \beta \Theta^{\prime \prime}(\Theta-\Phi)^{2}+\beta \Theta \Theta^{\prime 2}+0.17 \beta^{\prime \prime}(\Theta-\Phi)^{3}
\end{array}\right]
\end{array}\right] .
$$

Equations (15),(16), and (18) are a quasilinear system of the second order which describes the pendulum equations for this model. We aim to solve this system by a new procedure named the large parameter method [11] to get the approximated periodic solutions.
3.3. Approximate Periodic Solutions. In this section, we search the approximated periodic solutions for the case of nonresonance using the large parameter technique. Assuming the solutions for (15-18), the following is obtained:

$$
\begin{gathered}
\beta(\tau, \mu)=\beta_{0}+\mu^{-1} \beta_{1}+\mu^{-2} \beta_{2}+\ldots, \\
\Theta(\tau, \mu)=\Theta_{0}+\mu^{-1} \Theta_{1}+\mu^{-2} \Theta_{2}+\ldots, \\
\Phi(\tau, \mu)=\Phi_{0}+\mu^{-1} \Phi_{1}+\mu^{-2} \Phi_{2}+\ldots
\end{gathered}
$$

Substituting (18), (19), and (20) into (15), (16), and (18) and equating coefficients of same powers of $\mu$ in both sides, we get a system of nine equations which give the following solutions [3]:

$$
\begin{aligned}
\beta(\tau, \mu) & =\frac{b}{\omega_{n}^{2}-1} \sin \tau-\frac{\gamma}{\omega_{n}^{2}}-\mu^{-1} \frac{a^{2}}{h\left(\omega_{n}^{2}-4\right)} \cos 2 \tau+\ldots, \\
\Theta(\tau, \mu) & =-\frac{a}{h} \cos \tau+\mu^{-1}(a \sin 2 \tau-\Gamma \cos \tau)+\ldots, \\
\Phi(\tau, \mu) & =-\left(\frac{M a}{J}\right)\left[\frac{g}{\omega^{2}\left(\sigma^{2}-1\right)} \cos \tau-\frac{b}{2\left(\omega_{n}^{2}-1\right)\left(\sigma^{2}-4\right)} \sin 2 \tau\right]+\ldots
\end{aligned}
$$

The force of the spring will be

$$
F=k\left[d-\left(l+\frac{\gamma}{\omega_{n}^{2}}\right)+\frac{b}{\omega_{n}^{2}-1} \sin \tau-\mu \frac{a^{2}}{h\left(\omega_{n}^{2}-4\right)} \cos 2 \tau+\ldots\right] .
$$

\section{Numerical Considerations}

In this section, we treat the previously mentioned models by nine programs for obtaining both the analytical and the numerical solutions for different cases of the motions. We use the fourth-order Runge-Kutta method for obtaining the numerical solutions for systems of motions of the different problems. So, we obtain five tables of results and 33 figures for a description of the motions at different values of the pendulum parameters. These tables and figures describe the behavior of the motion and the influence of the different parameters on the solutions.

4.1. The Numerical Considerations of the First Model. In this section, we discuss the analytical and numerical solutions for the first model mentioned above. We compare these solutions, and we will discuss the maximum value of the angle $\Phi$. We divide the problem into the following cases.

4.1.1. The First Case $(\nu=0$ and $\mu=5000)$. Since $\nu=a / b=0$, then $a=0$, that is, the pendulum moves horizontally on a straight line of length $2 b$ [13]. From Table 1 , we note that the amplitude of the vibrations and the angular velocity decrease when $\Omega$ increases. Also, we note from the table that for every 
TABLE 1: $\varphi_{\max }$ of the first case $(\nu=0, \mu=5000)$.

\begin{tabular}{lcc}
\hline$\Omega$ & $\varphi_{\max }$ analytical & $\varphi_{\max }$ \\
\hline 2.1 & 0.2933488 & 0.2930785 \\
2.3 & 0.2331735 & 0.2331879 \\
2.5 & 0.1905422 & 0.1905665 \\
2.7 & 0.1590564 & 0.1590581 \\
2.8 & 0.146291 & 0.1463126 \\
3.1 & 0.1160335 & 0.1161796 \\
3.3 & 0.1010852 & 0.1011365 \\
3.5 & 0.08887673 & 0.08893137 \\
3.7 & 0.07879558 & 0.07887411 \\
4.1 & 0.06324854 & 0.06334555 \\
4.3 & 0.0571738 & 0.05724992 \\
4.5 & 0.05194685 & 0.05202674 \\
4.7 & 0.04741498 & 0.0474991 \\
5.2 & 0.03840205 & 0.03847147 \\
5.4 & 0.03551105 & 0.03557474 \\
\hline
\end{tabular}

value of $\Omega$ there is a great value for $\Phi$ (the angle between the pendulum rib and the vertical axis). This case is represented (see Figure 2).

At the initial moment $(\tau=0)$, the pendulum is suspended at point $\mathrm{O}$ and its rib is represented by the vector $\underline{O B}$, i.e., $(\Phi=0)$. The angle $\Phi$ increases counterclockwise until it reaches the maximum value when the suspension point is at $A(\tau=\pi / 2)$. At $\tau=\pi$ the suspension, the point returns to the initial position $O$. Then, the angle $\Phi$ increases in a clockwise direction until it reaches its maximum value at the point $A^{\prime}(\tau=3 \pi / 2)$. The suspension point will then be directed towards the point $\mathrm{O}$ until it reaches the initial position $(\tau=2 \pi)$. In this case, the graphical representations of analytical and numerical solutions appear (see Figures 3 and 4).

4.1.2. The Second Case $(\nu=1$ and $\mu=5000)$. In this case, $\nu=$ $a / b=1$ and then $a=b$; this means that the point of suspension of the pendulum moves on a circle of radius $a$. It is obvious from Table 2 that when the value of $\Omega$ increases, the angular velocity decreases and the amplitude of the vibrations decreases. The movement of the suspension point on the circumference of a circle can be illustrated (see Figure 5). At the initial moment $(\tau=0)$, the point of suspension of the pendulum is at $S$ and its rib is represented by the vector $\underline{S B}$, i.e., $(\Phi=0)$. Then, the pendulum's suspension point moves counterclockwise towards point $A$. The angle $\Phi$ increases counterclockwise until it reaches the maximum value when the suspension point is at $A(\tau=\pi / 2)$. When the suspension point reaches the point $S^{\prime}(\tau=\pi)$, the angle $\Phi$ becomes zero and the pendulum rib becomes vertical again. Then, the angle $\Phi$ increases until it reaches its maximum value at the point $A^{\prime}(\tau=3 \pi / 2)$. After that, the suspended point will be directed towards point $S$ until it reaches the primary position $(\tau=2 \pi)$ and completes the period. As for this case, the graphical representations for both analytical and numerical solutions appear (see Figures 6 and 7).

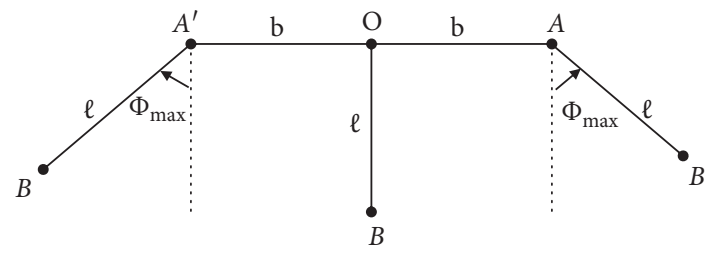

FIgURE 2: Description of the pendulum motion for case (i) in Section 4.1.

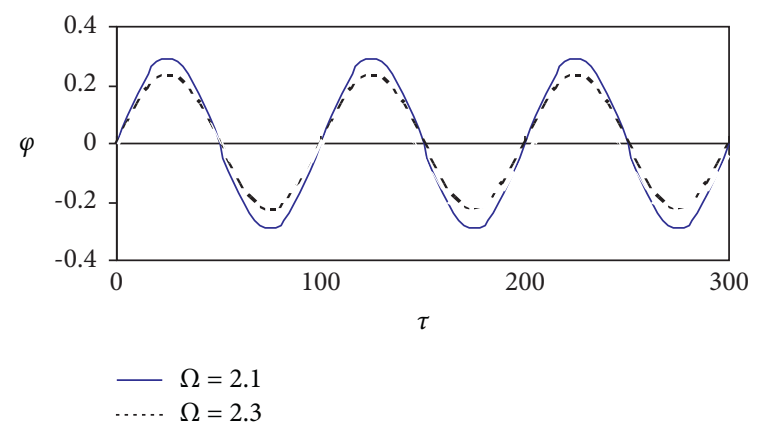

Figure 3: The analytical solutions for case (i) in Section 4.1.

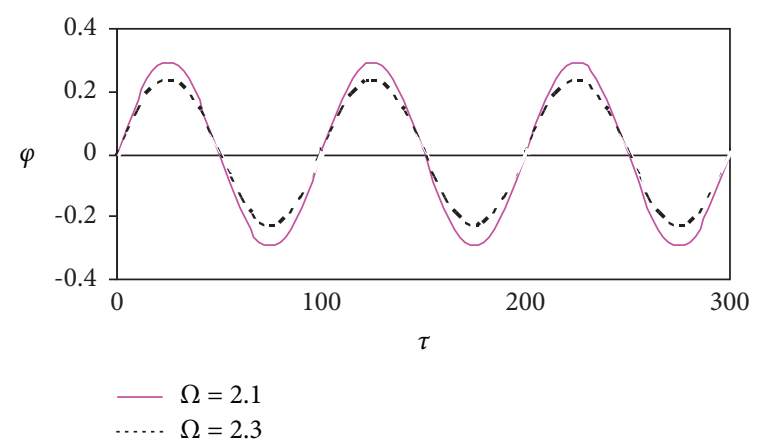

Figure 4: The numerical solutions for case (i) in Section 4.1. 
TABLE 2: $\varphi_{\max }$ of the second case $(\nu=1, \mu=5000)$.

\begin{tabular}{lcc}
\hline$\Omega$ & $\varphi_{\max }$ analytical & $\varphi_{\max }$ numerical \\
\hline 2.1 & 0.3213152 & 0.3233396 \\
2.3 & 0.2336605 & 0.2339807 \\
2.5 & 0.19085 & 0.1909746 \\
2.7 & 0.159339 & 0.1591531 \\
2.8 & 0.1466192 & 0.1466426 \\
3.1 & 0.1156941 & 0.1158647 \\
3.3 & 0.1010075 & 0.1010626 \\
3.5 & 0.08884358 & 0.0889034 \\
3.7 & 0.07877824 & 0.07885911 \\
4.1 & 0.06324223 & 0.06336687 \\
4.3 & 0.05716965 & 0.0572462 \\
4.5 & 0.05194401 & 0.05202422 \\
4.7 & 0.04741298 & 0.0474967 \\
5.2 & 0.03840113 & 0.03846995 \\
5.4 & 0.03551035 & 0.03557364 \\
\hline
\end{tabular}

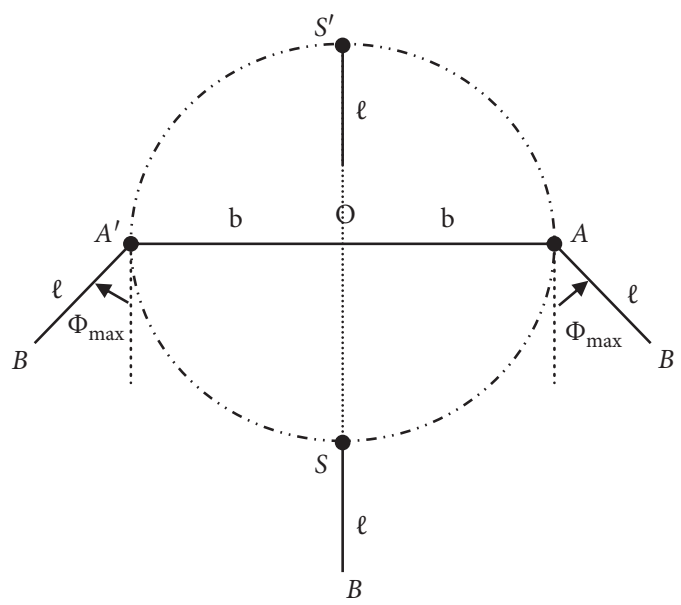

FIGURE 5: Description of the pendulum motion for case (ii) in Section 4.1.

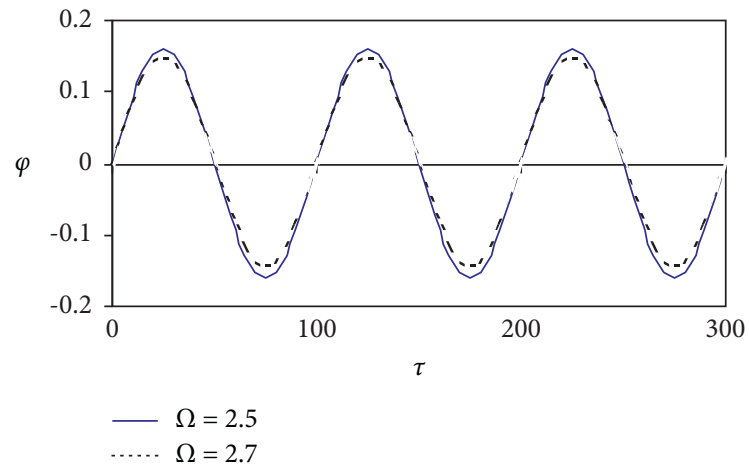

Figure 6: The analytical solutions for case (ii) in Section 4.1.

4.1.3. The Third Case $(\nu=1.5$ and $\mu=5000)$. It is clear from Table 3 that the amplitude of the vibrations decreases when the value of $\Omega$ increases and thus the angular velocity decreases. The graphical representations of the analytical and numerical solutions are shown (see Figures 8 and 9).

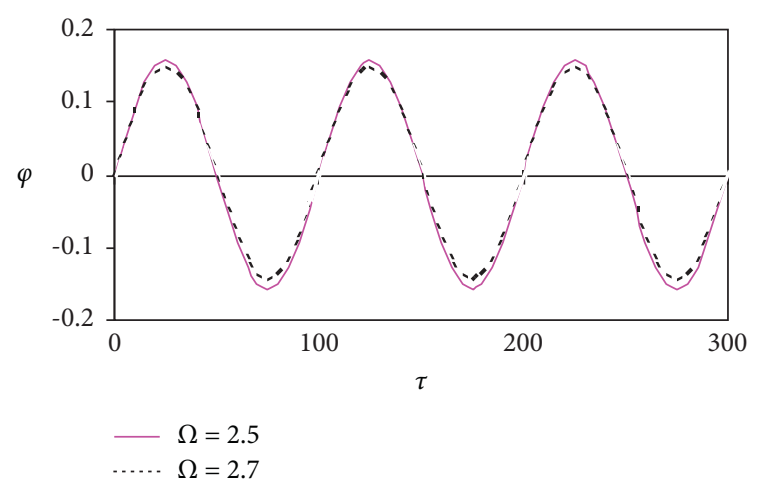

FIGURE 7: The numerical solutions for case (ii) in Section 4.1.

TABle 3: $\varphi_{\max }$ of the third case $(\nu=1.5, \mu=5000)$.

\begin{tabular}{lcc}
\hline$\Omega$ & $\varphi_{\max }$ analytical & $\varphi_{\max }$ numerical \\
\hline 2.1 & 0.3433021 & 0.3466265 \\
2.3 & 0.2382122 & 0.2389854 \\
2.5 & 0.1912348 & 0.1914796 \\
2.7 & 0.1596922 & 0.1597867 \\
2.8 & 0.1470294 & 0.1470511 \\
3.1 & 0.1152698 & 0.1154751 \\
3.3 & 0.1009105 & 0.1009705 \\
3.5 & 0.08880214 & 0.08886883 \\
3.7 & 0.07875657 & 0.07884256 \\
4.1 & 0.06323432 & 0.06333476 \\
4.3 & 0.05716445 & 0.0572428 \\
4.5 & 0.05194046 & 0.05202207 \\
4.7 & 0.04741048 & 0.04749489 \\
5.2 & 0.03839997 & 0.03846891 \\
5.4 & 0.03550947 & 0.03557289 \\
\hline
\end{tabular}

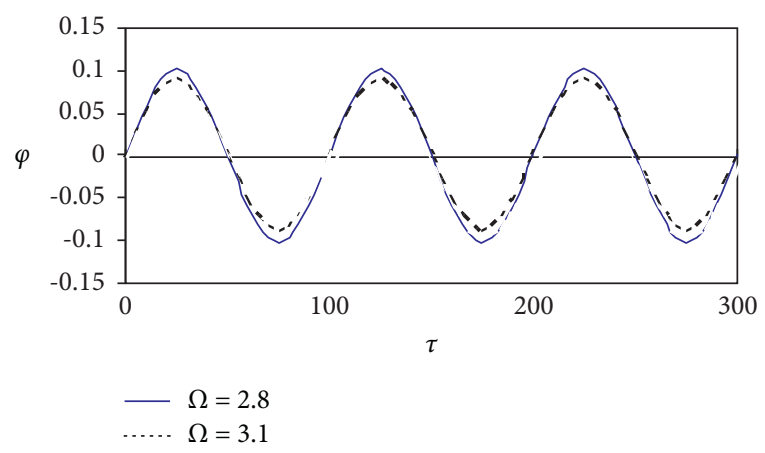

FIGURE 8: The analytical solutions for case (iii) in Section 4.1.

4.1.4. The Fourth Case $(\Omega=1.5$ and $\mu=1250)$. Table 4 shows the analytical and numerical solutions of different values of $v$ belonging to the period $[0,1]$. From Table 4 , we conclude that the higher the value $\mu$ makes the smaller the amplitude of the vibrations. The graphical representations for this case are obtained (see Figures 10 and 11).

4.1.5. The Fifth Case $(\Omega=2.5$ and $\mu=1250)$. Table 5 gives the analytical and numerical solutions for different values of $\nu$ belonging to the period $[0,1]$. We conclude that the higher 


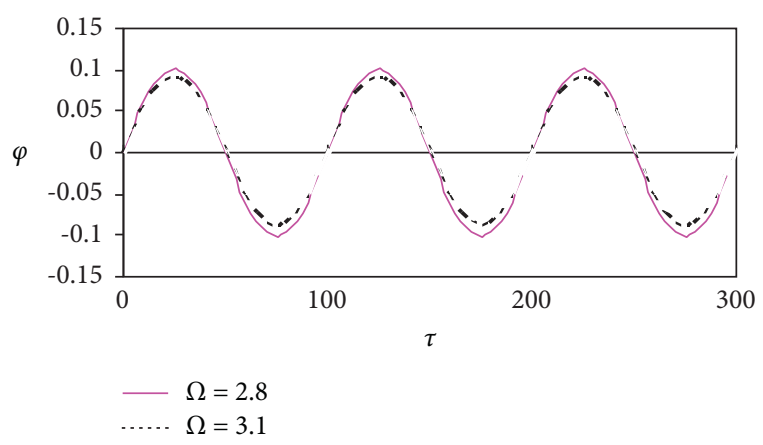

FIgURE 9: The numerical solutions for case (iii) in Section 4.1.

TABLE 4: $\varphi_{\max }$ of the fourth case $(\Omega=1.5, \mu=1250)$.

\begin{tabular}{lcc}
\hline$\nu$ & $\varphi_{\max }$ analytical & $\varphi_{\max }$ numerical \\
\hline 0 & 0.8000296 & 0.8000393 \\
0.1 & 0.8000292 & 0.800032 \\
0.2 & 0.800028 & 0.8000173 \\
0.3 & 0.8000258 & 0.7998232 \\
0.4 & 0.8000228 & 0.7998127 \\
0.5 & 0.8000191 & 0.799799 \\
0.6 & 0.8000144 & 0.799782 \\
0.7 & 0.8000089 & 0.7997616 \\
0.8 & 0.8000026 & 0.7997379 \\
0.9 & 0.7999954 & 0.7997114 \\
1 & 0.7999873 & 0.7996807 \\
\hline
\end{tabular}

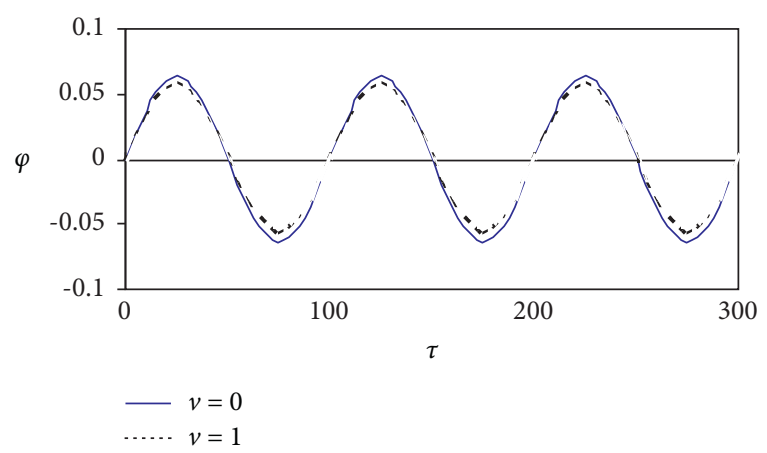

FIgURE 10: The analytical solutions for case (iv) in Section 4.1.

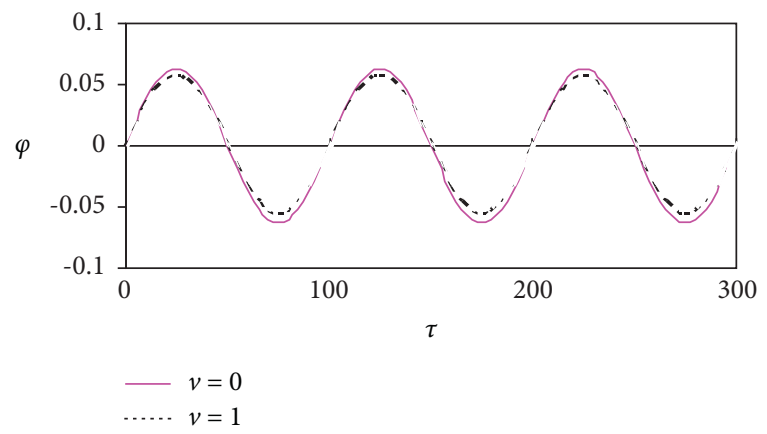

FIgURE 11: The numerical solutions for case (iv) in Section 4.1.
TABLE 5: $\varphi_{\max }$ of the fifth case $(\Omega=2.5, \mu=1250)$.

\begin{tabular}{lcc}
\hline$\nu$ & $\varphi_{\max }$ analytical & $\varphi_{\max }$ numerical \\
\hline 0 & 0.1904803 & 0.190602 \\
0.1 & 0.1904805 & 0.1906011 \\
0.2 & 0.1904811 & 0.1906013 \\
0.3 & 0.1904821 & 0.1906015 \\
0.4 & 0.1904834 & 0.1906024 \\
0.5 & 0.1904851 & 0.1906038 \\
0.6 & 0.1904873 & 0.1906058 \\
0.7 & 0.1904897 & 0.1906083 \\
0.8 & 0.1904926 & 0.1906113 \\
0.9 & 0.1904959 & 0.1906149 \\
1 & 0.1904996 & 0.1906189 \\
\hline
\end{tabular}

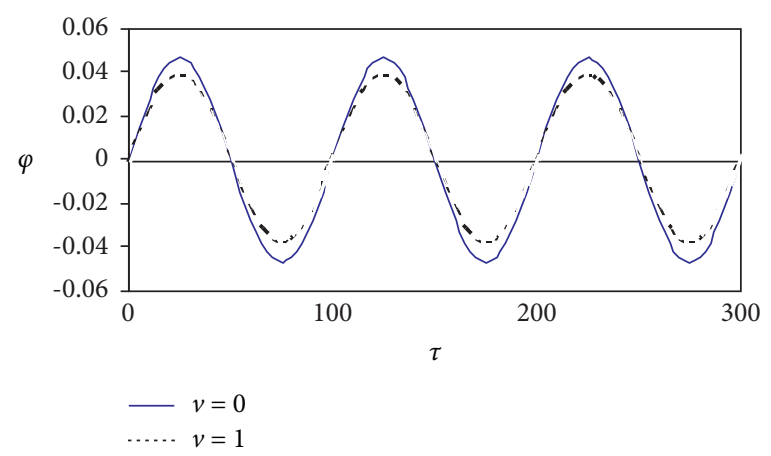

Figure 12: The analytical solutions for case (v) in Section 4.1.

the value $v$, the slower the amplitude of the vibrations will increase. From Table 5, we find that the difference between numerical and analytical results is very small and can be neglected. Moreover, the graphic representations of this case are shown (see Figures 12 and 13).

4.2. The Numerical Considerations of the Second Model. This section will be devoted to verifying the accuracy of the analytical solutions resulting in the second model mentioned above by using computer programs. These solutions will be represented graphically in several cases as follows.

4.2.1. The First Case $(b=0$ and $\mu=2500)$. Since $b=0$, this means that the movement of the pendulum is horizontal along its longitude $2 a$. This is evident from the graphical representations of the analytical solutions shown through the graphs (see Figures 14-16). In this case, we note the stability of the solutions that we obtained as evidence (see Figures 17-19).

4.2.2. The Second Case $(a=b$ and $\mu=2500)$. In this case, the point of suspension of the pendulum moves over a circle of radius $a$. We obtain the graphic representations of the analytical solutions in a suitable manner of the case (i) (see Figures 20-22). We note the stability of solutions in this case, as evidenced (see Figures 23-25). 


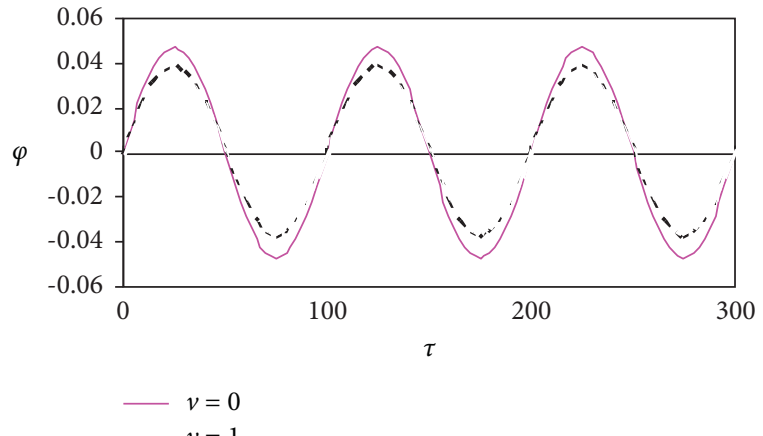

FIgURE 13: The numerical solutions for case (v) in Section 4.1.

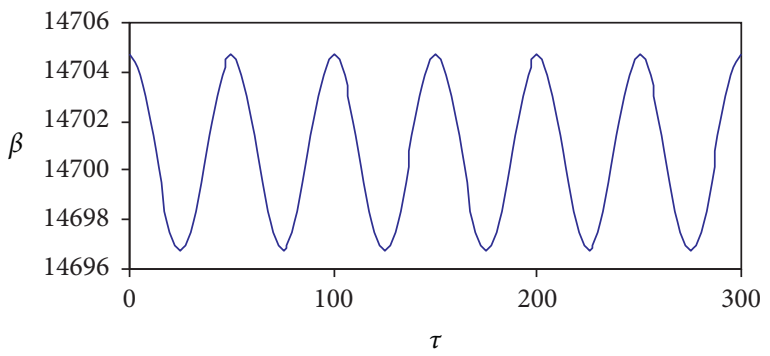

Figure 14: The analytical solution $\beta$ for case (i) in Section 4.1.

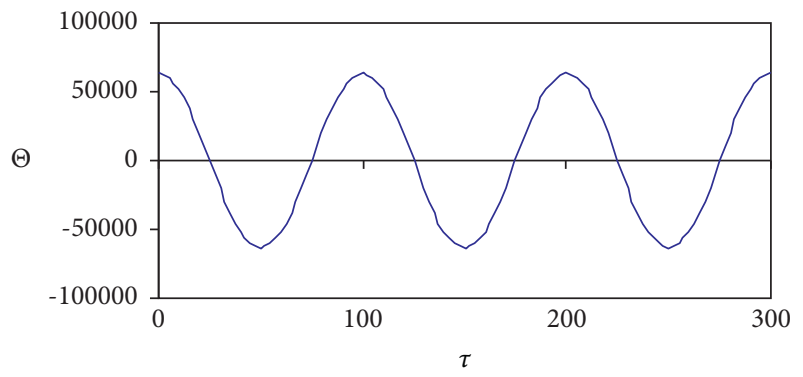

FIgURe 15: The analytical solution $\Theta$ for case (i) in Section 4.2.

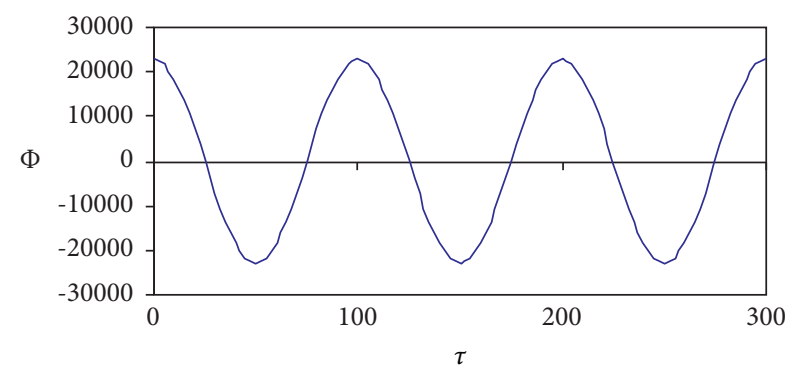

FIgURE 16: The analytical solution $\Phi$ for case (i) in Section 4.2.

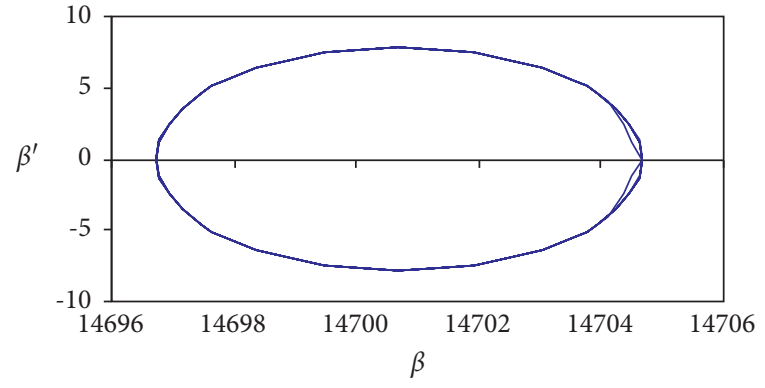

FIgURE 17: The stability phrase $\beta^{\prime}$ and $\beta$ for case (i) in Section 4.2.

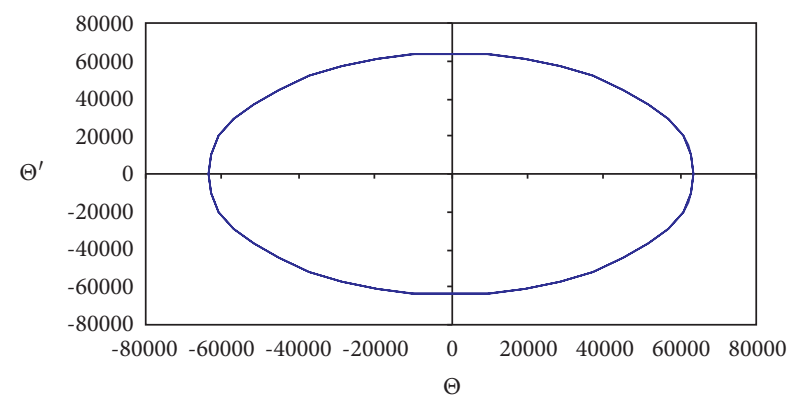

FIgURE 18: The stability phrase $\Theta^{\prime}$ and $\Theta$ for case (i) in Section 4.2.

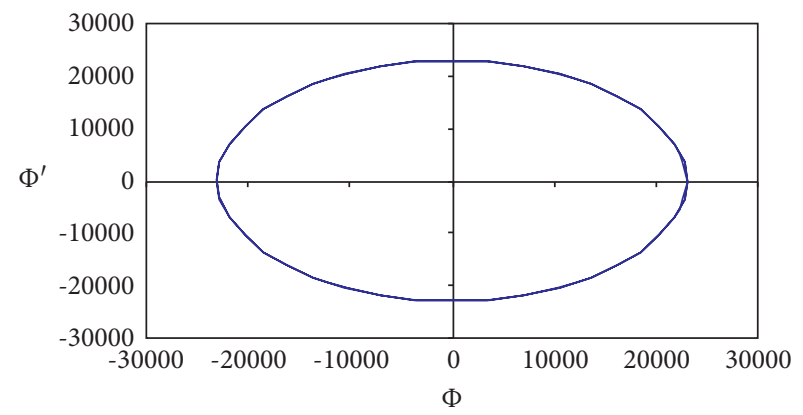

Figure 19: The stability phrase $\Phi^{\prime}$ and $\Phi$ for case (i) in Section 4.2.

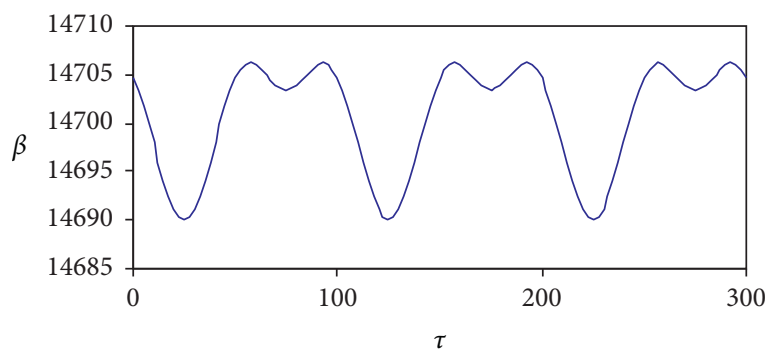

Figure 20: The analytical solution $\beta$ for case (ii) in Section 4.2. 


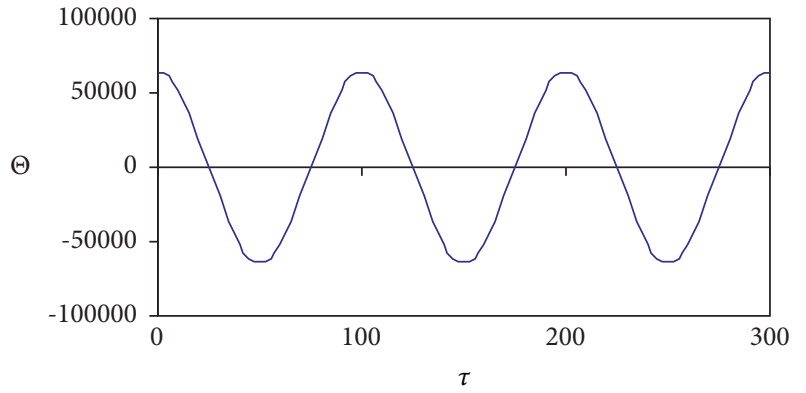

Figure 21: The analytical solution $\Theta$ for case (ii) in Section 4.2.

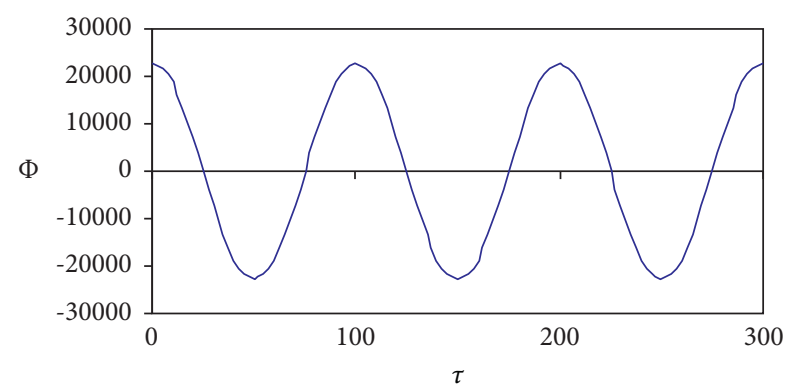

FIgURE 22: The analytical solution $\Phi$ for case (ii) in Section 4.2.

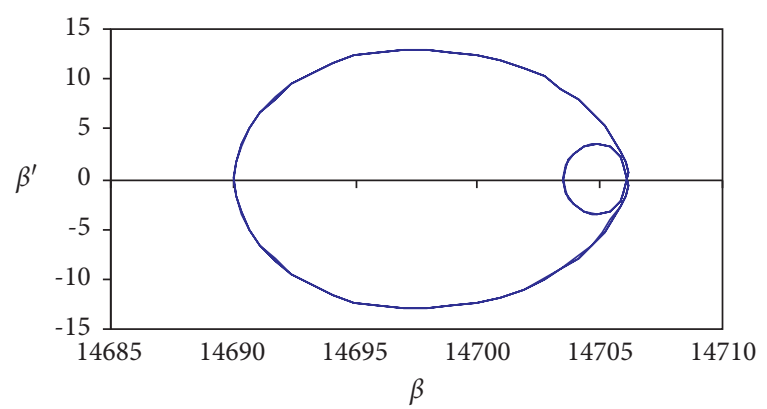

FIgURE 23: The stability phrase $\beta^{\prime}$ and $\beta$ for case (ii) in Section 4.2.

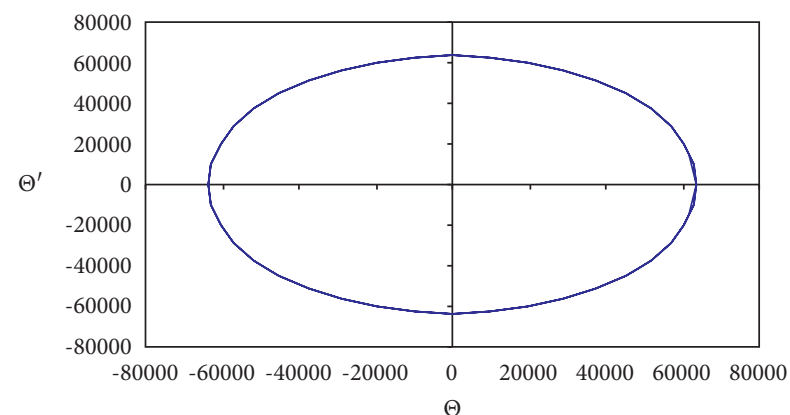

FIgURE 24: The stability phrase $\Theta^{\prime}$ and $\Theta$ for case (ii) in Section 4.2.

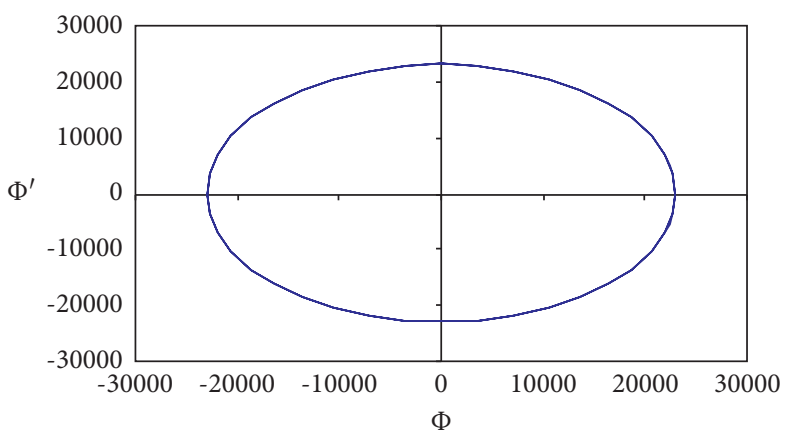

FIgUre 25: The stability phrase $\Phi^{\prime}$ and $\Phi$ for case (ii) in Section 4.2.

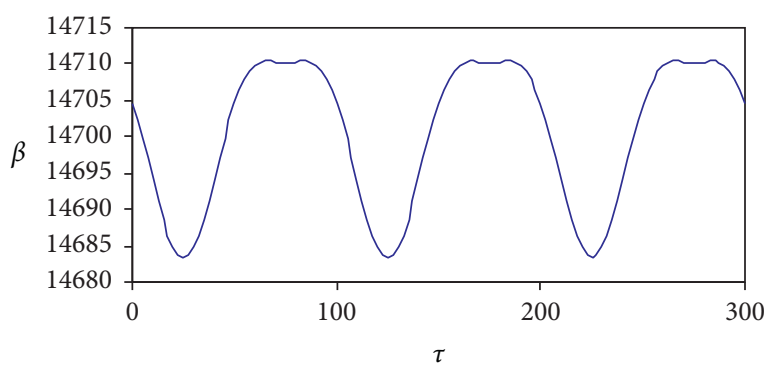

FIgURE 26: The analytical solution $\beta$ for case (iii) in Section 4.2.

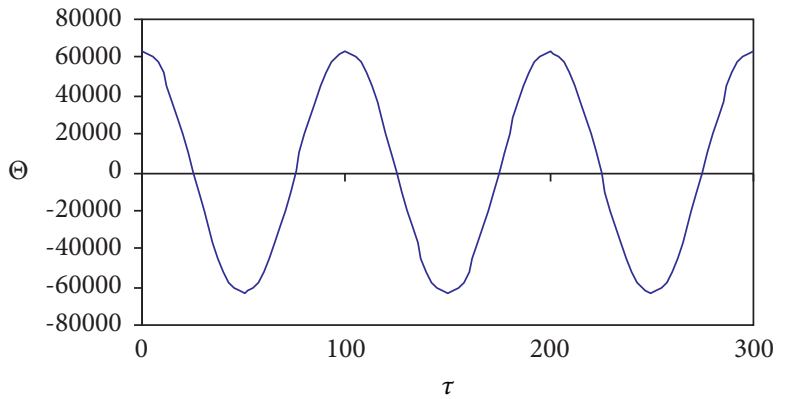

FIgURE 27: The analytical solution $\Theta$ for case (iii) in Section 4.2.

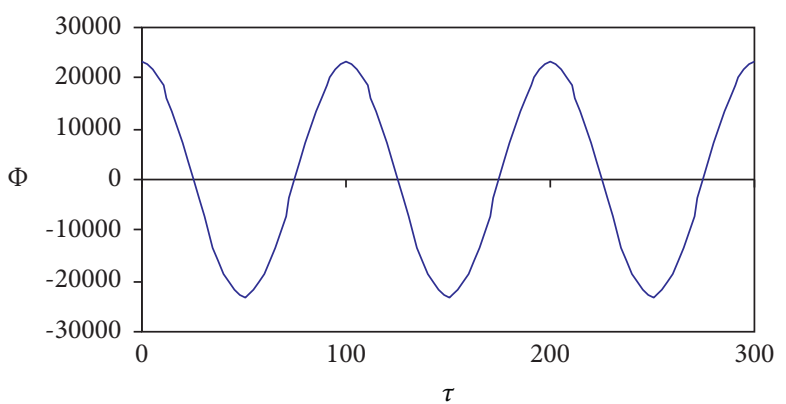

FIgURE 28: The analytical solution $\Phi$ for case (iii) in Section 4.2. 


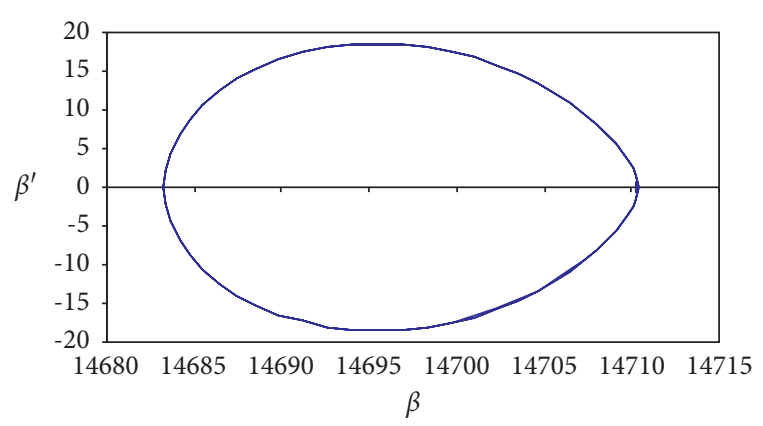

Figure 29: The stability phrase $\beta^{\prime}$ and $\beta$ for case (iii) in Section 4.2.

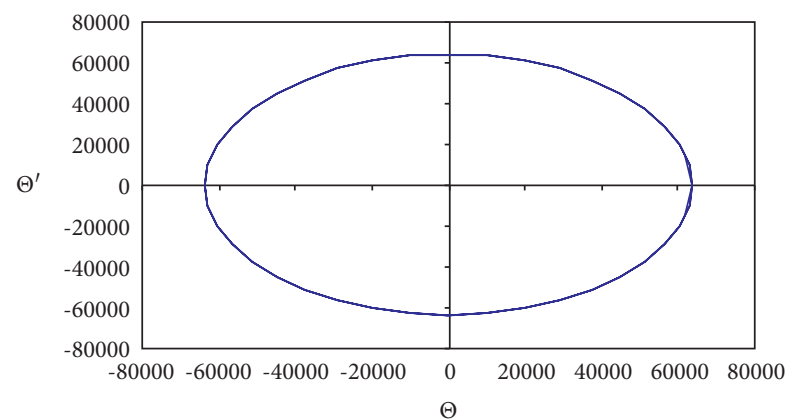

Figure 30: The stability phrase $\Theta^{\prime}$ and $\Theta$ for case (iii) in Section 4.2.

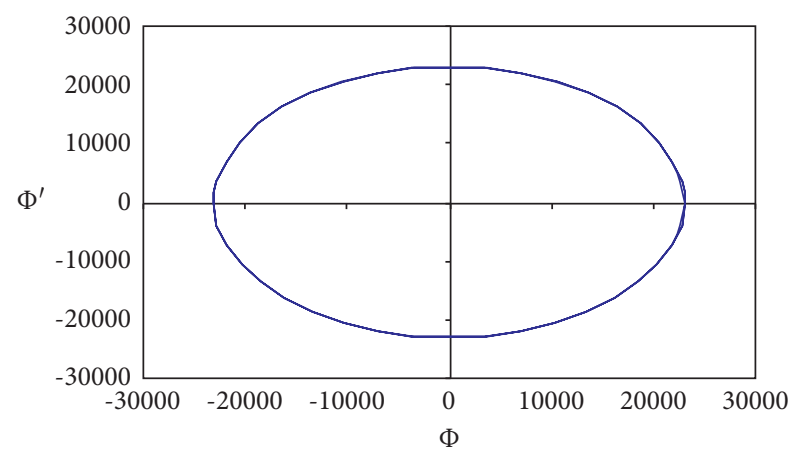

Figure 31: The stability phrase $\Phi^{\prime}$ and $\Phi$ for case (iii) in Section 4.2.

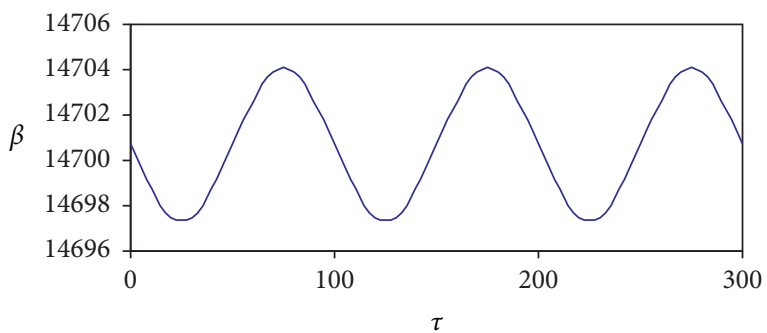

Figure 32: The analytical solution $\beta$ for case (iv) in Section 4.2.

4.2.3. The Third Case $(b=2 a$ and $\mu=2500)$. In this case, the pendulum's suspension point moves on the ellipse of its largest and minimum axial as $a$ and $b$, respectively. Concerning this case, the graphic representations of the analytical solutions appear suitably for case (i) (see

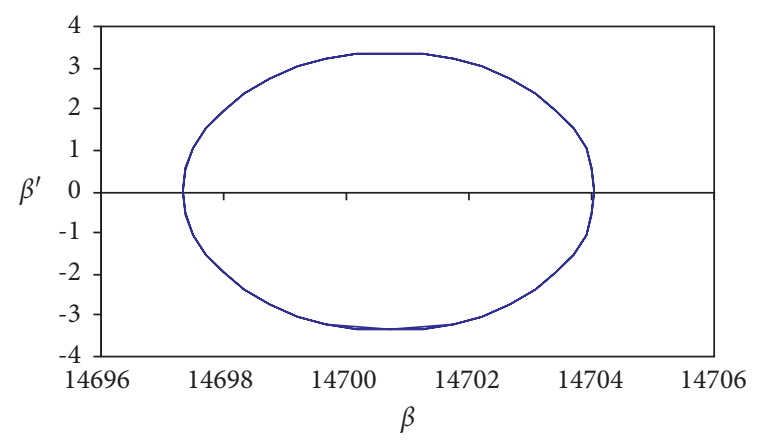

FIgURE 33: The stability phrase $\beta^{\prime}$ and $\beta$ for case (iv) in Section 4.2.

Figures 26-28). We note the stability of solutions in this case, as evidenced (see Figures 29-31).

4.2.4. The Fourth Case $(a=0)$. The pendulum, in this case, moves vertically along a vertical line along its length, and the graphic representations of the analytical solutions are represented (see Figure 32). In this case, we note the stability of the solutions as shown (see Figure 33). In this case, we deduce that $\Theta(\tau)=\Phi(\tau)=0$.

\section{Conclusions}

Two new models have been introduced for the movement of the pendulum in the presence of new primary conditions that are not previously defined. Poincare's method fails to solve these problems in the presence of the new condition so we must search for a new technique that matches these changes. A large parameter was defined to achieve a large parameter method for solving this problem under the new assumptions. The equations for the motility of the models are deduced and solved using the large parameter method for obtaining the solutions analytically. The fourth-order Ronge-Kutta numerical method is presented for solving the system of equations numerically through computer programs. Also, numerical and analytical solutions were compared by 5 tables and 33 graphs. It turned out that the analytical solutions conform to the numerical solutions, which proves the validity of the serious methods used in the solutions. The solutions stabilities are given by the phase diagrams procedure. This paper is a generalization of many previous works. The two models are classified into nine cases depending on the parameters of the motion. From this, we conclude that the cases studied were implemented in research $[6,7,10,13]$. There are generalized cases of the pendulum movement because of introducing the coherent body instead of the particle as well as the movement on an ellipse instead of moving on a circle and taking a flexible wire instead of a string. The previous solutions are obtained as special cases of solutions in this paper. We also conclude the following points:

(1) The approximate periodic solutions are obtained using the large parameter method because Poincare's technique is failed in this case 
(2) The amount of angular velocity in the case of nonresonant vibrations must take no integer values to avoid the singularity in the solution

(3) The approximate periodic solutions were obtained in terms of periodic functions.

\section{Data Availability}

Data sharing is not applicable to this article as no datasets were generated or analyzed during the current study

\section{Conflicts of Interest}

The authors declare that there are no conflicts of interest.

\section{Authors' Contributions}

AI carried out the design of the article and the immunoassays, participated in the sequence alignment, analyzed and interpreted the methods, performed the results, concluded the study, was a major contributor in writing the manuscript, and conceived and coordinated the study. HA carried out the numerical analysis, participated in writing and revising the sequence alignment, drafted the manuscript, participated in the design of the study, and performed the numerical analysis. All authors read and approved the final manuscript.

\section{Acknowledgments}

The authors would like to thank the Deanship of Scientific Research at Umm Al-Qura University for supporting this work by Grant Code: (22UQU4240002DSR01).

\section{References}

[1] F. A. El-Barki, A. I. Ismail, M. O. Shaker, and T. S. Amer, "On the motion of the pendulum on an Ellipse," Zeitschrift für Angewandte Mathematik und Mechanik, vol. 79, no. 1, pp. 65-72, 1999.

[2] S. J. Liao, "A second-order approximate analytical solution of a simple pendulum by the process analysis method," Journal of Applied Mechanics, vol. 59, no. 4, pp. 970-975, 1992.

[3] A. I. Ismail, "Relative periodic motion of a rigid body pendulum on an ellipse," Journal of Aerospace Engineering, vol. 22, no. 1, 2009.

[4] W. S. Amer, M. A. Bek, and M. K. Abohamer, "On the motion of a pendulum attached with tuned absorber near resonances," Results in Physics, vol. 11, pp. 291-301, 2018.

[5] T. S. Amer, B. Ma, and I. S. Hamada, "On the motion of harmonically excited spring pendulum in the elliptic path near resonances," Advances in Mathematical Physics, 2016.

[6] N. V. Stoianov, "On the relative periodic motions of a pendulum," Journal of Applied Mathematics and Mechanics, vol. 28, no. 1, pp. 160-163, 1964.

[7] A. Werner and C. J. Eliezer, "The lengthening pendulum," Journal of the Australian Mathematical Society, vol. 9, no. 3-4, pp. 331-336, 1969.

[8] A. H. Nayfeh, "A perturbation method for treating nonlinear oscillation problems," Journal of Mathematics and Physics, vol. 44, no. 1-4, pp. 368-374, 1965.
[9] W. K. Tso and K. G. Asmis, "Parametric excitation of a pendulum with bilinear hysteresis," Journal of Applied Mechanics, vol. 37, no. 4, pp. 1061-1068, 1970.

[10] B. A. Schmidt, "The radially flexible pendulum subjected to a high-frequency excitation," Journal of Applied Mechanics, vol. 50, no. 2, pp. 443-448, 1983.

[11] A. I. Ismail, "Solving a problem of rotary motion for a heavy solid using the large parameter method," Advances in Astronomy, vol. 2020, Article ID 2764867, 7 pages, 2020.

[12] T. Van Baak, "A new and wonderful pendulum period equation (PDF)," Horological Science Newsletter, vol. 2013, no. 5, pp. 22-30, 2013.

[13] K. Ochs, "A comprehensive analytical solution of the nonlinear pendulum," European Journal of Physics, vol. 32, no. 2, pp. 479-490, 2011. 